\title{
ESTUDO COMPARATIVO DA AÇÃO ANTI-HELMÍNTICA DA BATATA DE PURGA (Operculina hamiltonii) E DO MELÃO DE SÃO CAETANO (Mormodica charantia) EM CAPRINOS (Capra hircus) NATURALMENTE INFECTADOS ${ }^{1}$
}

\author{
Comparative study of the anti-helminthic action of the potato of purges (Operculina hamiltonii) and \\ the cantaloups of São Caetano (Mormodica charatia) in naturally infected goats (Capra hircus) ${ }^{1}$
}

\author{
Luciano de Brito-Junior², Maria Luana Cristiny Rodrigues Silva ${ }^{3}$, Francisco Heitor de Lima ${ }^{3}$, \\ Ana Célia Rodrigues Athayde ${ }^{4}$, Wilson Wouflan Silva ${ }^{4}$, Onaldo Guedes Rodrigues ${ }^{4}$
}

\begin{abstract}
RESUMO
As helmintoses gastrintestinais ocupam lugar de destaque na produção de pequenos ruminantes como um fator limitante e o seu controle vem sendo realizado por meio do uso indiscriminado de anti-helmínticos favorecendo o surgimento da resistência a medicamentos. Com o objetivo de comparar a ação anti-helmíntica das plantas Operculina hamiltonii (batata de purga), Marmodica charantia (Melão de São Caetano) e do moxedctin a $0,2 \%$ sobre as infecções helminticas naturais de caprinos, foram utilizados 40 caprinos, sem raça definida, fêmeas, com idade entre seis e 12 meses, naturalmente infectados, separados em quatro grupos: o GRUPO 1 (G1) - animais controle negativo tratados com água destilada; o GRUPO $2(\mathrm{G} 2)$ - animais tratados com o extrato alcoólico da $O$. hamiltonii; o GRUPO 3 (G3) - animais tratados com o extrato alcoólico da M. charantia e no GRUPO 4 (G4) - animais controle positivo tratados com moxidectina $0,2 \%$. Todos os grupos receberam os tratamentos por três dias consecutivos. As amostras fecais foram coletadas nos dias 0, 30 e 60 após tratamento, para a realização da contagem de ovos por grama de fezes (OPG) e larvacultura. Para avaliar o efeito dos tratamentos, aplicou-se o teste de redução na contagem de ovos por grama de fezes (RCOF). A RCOF do G2, G3 e G4 foram de 63 e 90; 40 e 40; 100 e 100\%, respectivamente para 30 e 60 dias pós-tratamento respectivamente. Durante o período de estudo, observou-se que todas as amostras coletadas estavam positivas para larvas de helmintos da superfamília Trichostrongyloidea, com exceção para o G4, nos dias 30 e 60 pós-tratamento. O Haemonchus foi o parasita mais prevalente nas coproculturas.
\end{abstract}

Termos para indexação: Etnoveterinária, fitoterapia, helmintoses, caprinocultura.

\section{ABSTRACT}

Gastrintestinal helminthosis occupy a place of prominence in the production of small ruminants as an edge factor. Its control has been carried through the indiscriminate use of anti-helminthic products in favor of the appearing resistance to the medicines. With the objective to compare the anti-helminthic action of the Operculina hamiltonii plants (potato of purges), Marmodica charantia (Cantaloups of Sao Caetano) and of moxedctin at $0.2 \%$ about the natural helminthic infections of goats, there had been used 40 goats, without defined race, females, with age between six and 12 months, naturally infected, separated in four groups: GROUP 1 (G1) negative control animals treated with distilled water; GROUP $2(\mathrm{G} 2)$ - animal treated with the alcoholic extract of $O$. hamiltonii; GROUP 3 (G3) - animal treated with the alcoholic extract of $M$. charantia and GROUP 4 (G4) - positive control animals treated with moxidectina at $0.2 \%$. All of them had received the treatments for three days in a row. The feces samples were collected at: 0,30 and 60 days after treatment, for the accomplishment of the egg counting per gram of excrements (EPG) and larvae culture. To evaluate the effect of the treatments, a reduction on the egg counting per gram of excrements (RECE) was applied. The RECE of the G2, G3 and G4 were: 63 and 90; 40 and 40; 100 and 100\%, respectively for 30 and 60 days post-treatment, respectively. During the period of study it was observed that all collected samples were positive for larvae of helminths of the superfamily Trichostrongyloidea, except for the G4, throughout 30-60 days and post-treatment. The Haemonchus was the most prevalent parasite in the culture of feces.

Index terms: Ethnoveterinary, fithotherapy, helminthosis, caprinoculture (goat farming).

(Recebido em 25 de maio de 2009 e aprovado em 10 de fevereiro 2010)

\section{INTRODUÇÃO}

O Brasil possui aproximadamente 12,6 milhões de cabeças de caprinos, correspondendo ao $11^{\circ}$ maior rebanho do mundo. O nordeste é a região mais representativa do Brasil com relação ao número de caprinos, possuindo um rebanho de cerca de 8,9 milhões caprinos, compreendendo aproximadamente a $93 \%$ do rebanho nacional (ANUALPEC, 2002).

Os caprinos constituem uma fonte de proteína de origem animal, o que torna a caprinocultura uma das

'Parte da dissertação defendida no Programa de Pós-Graduação em Zootecnia pela Universidade Federal de Campina Grande/UFCG ${ }^{2}$ Universidade Federal de Campina Grande/UFCG - Unidade Acadêmica de Ciências Biológicas/UACB - Campus de Patos - Avenida Universitária, s/n Santa Cecília - 58708-110 - Patos, PB - Ibritojunior@hotmail.com

${ }^{3}$ Universidade Federal de Campina Grande/UFCG - Patos, PB

${ }^{4}$ Universidade Federal de Campina Grande/UFCG - Centro de Saúde e Tecnologia Rural/CSTR - Unidade Acadêmica de Ciências Biológicas/UACB - Patos, PB 
atividades importantes, do ponto de vista socioeconômico, tanto no Brasil como no mundo onde existe a exploração da espécie. Nas criações maiores e mais tecnificadas, a cabra aparece como geradora de empregos, permitindo a uma parcela da população a ter o seu sustento garantido por via direta (trabalho na criação), bem como por via indireta (nas queijarias, fábricas de couro) (VIEIRA et al., 1998; RODRIGUES et al., 2007).

O parasitismo é causa primária da redução do potencial produtivo animal, sendo responsável por altas perdas econômicas, em decorrência de crescimento retardado, perda de peso, diminuição da ingestão de alimentos, redução na produção de leite, baixa fertilidade e nos casos de infecções maciças, altas taxas de mortalidade (GOPALet al., 1999; MOLENTO, 2004).

Só com tratamento de infecções por Haemonchus, o Quênia e a África do Sul, apresentam perdas anuais entre US\$ 26 e 45 milhões. Também foram calculados custos associados com o controle desses parasitos na Índia, aonde chega à cifra de US\$ 103 milhões (MCLEOD, 2004; KRECEK; WALLER, 2006).

Os principais gêneros parasitas de caprinos são: Haemonchus, Trichostrongylus, Strongylus, Moniezia, Cooperia, Oesophagostomum, Skrjabinema, Trichuris e Cysticercus.

Surtos epizoóticos de haemoncose e strongiloidose caprina diagnosticados no semiárido paraibano vêm aumentando os índices de morbidade e mortalidade do efetivo caprino, comprometendo a produtividade do sistema (RODRIGUES et al., 2007).

O controle das parasitoses gastrintestinais de caprinos é realizado principalmente com o uso de antihelmínticos sintéticos comerciais. Embora sejam utilizados em todas as espécies domésticas, o maior mercado é certamente aquele destinado aos ruminantes (URQUHART, 1996). No entanto, a utilização incorreta e indiscriminada desses produtos tem provocado o surgimento do fenômeno da resistência a anti-helmínticos (RA) (VIEIRA; CAVALCANTE, 1998; RODRIGUES et al., 2007; CEZAR et al., 2008). Esse fator, aliado ao manejo inadequado, tem estimulado pesquisadores e criadores a buscarem novos meios alternativos no controle de helmintos em pequenos ruminantes.

Roeder (1988) refere-se à importância do emprego de plantas medicinais nas enfermidades dos rebanhos no semiárido do Nordeste brasileiro e sugere a intensificação de pesquisas com fitoterápicos.

Araújo-Lima et al. (2002), em trabalho de difusão do uso de plantas medicinais com produtores de caprinos da região de Patos - PB, indicaram como plantas medicinais com ação sobre vermes o melão de São Caetano
(Mormodica charantia), a batata de purga (Operculina hamiltonii) e a semente da abóbora (Cucurbita pepo).

Almeida et al. (2003), utilizando os extratos aquosos das folhas de capim santo (C. citratus) e o Capim-Açu (Digitaria insularis) no tratamento in vitro de nematóides gastritestinais de caprinos, observaram uma redução superior a 95\% do número de larvas da superfamília Strongyloidea, na concentração de $224 \mathrm{mg} / \mathrm{ml}$ para o extrato de Capim-santo e entre 355,2 e 138,75 mg/ml para o extrato de Capim-açu.

Araújo-Lima et al. (2002), administrando dose oral de 90g/animal do melão de São Caetano (M. charantia), verificaram a redução no número de OPG em caprinos SRD infectados naturalmente, na região semiárida da Paraíba.

Conduziu-se este estudo, com o objetivo de avaliar a ação anti-helmíntica da batata de purga (Operculina hamiltonii) e do melão de São Caetano (Mormodica charantia), plantas popularmente usadas no semiárido paraibano.

\section{MATERIAL E MÉTODOS}

\section{Local de realização do experimento}

O experimento foi desenvolvido por um período de 60 dias, em propriedades do sistema produtivo de caprinos do semiárido e nos Laboratórios de Doenças Parasitárias dos Animais Domésticos e de Ciências Químicas Biológicas da Unidade Acadêmica de Medicina Veterinária (UAMV) do Centro de Saúde e Tecnologia Rural (CSTR) da Universidade Federal de Campina Grande (UFCG), Campus de Patos - PB.

\section{Animais}

Foram utilizados 40 caprinos, sem raça definida, fêmeas, com idade entre seis e doze meses de vida, com peso de 14 a $18 \mathrm{~kg}$. Os animais não precisaram sofrer adaptação em função de já estarem familiarizados com o ambiente utilizado. $\mathrm{O}$ sistema de marcação foi brincagem. Os animais foram examinados previamente para diagnóstico do parasitismo natural por helmintos gastrintestinais.

\section{Coleta de Fezes}

As amostras fecais individuais foram obtidas diretamente da ampola retal com auxílio de tubos de ensaio lubrificados com glicerina líquida. As amostras de fezes foram identificadas, posteriormente acondicionadas em caixas térmicas e refrigeradas com gelo até o seu processamento.

\section{Exames realizados}

Os exames realizados foram à contagem do número de ovos por grama de fezes (OPG) pela técnica descrita por 
Gordon e Whitlok (1939) e coproculturas (ROBERTS; O'SULLIVAN, 1950) que foram realizadas em pool, misturando-se as amostras fecais dos animais de cada grupo experimental, identificando-se um mínimo de 100 larvas de terceiro estágio (KEITH, 1953). Somente após o diagnóstico da infecção helmíntica, os animais foram submetidos aos tratamentos experimentais. Os exames foram realizados nos dias 0,30 e 60 , do período experimental.

\section{Preparo e Manipulação das Plantas}

As amostras das plantas utilizadas foram todas submetidas aos mesmos procedimentos laboratoriais. Após coleta e identificação botânica das partes indicadas no estudo etnofarmacológico e posterior herbarização, as folhas do melão de São Caetano e da bata de purga, foram higienizadas e, em seguida, picadas em triturador industrial, pesadas em balança eletrônica de precisão, para separação de alíquotas iguais e, por fim, armazenadas em bolsas de tecido não tecido (TNT). Em seguida, as amostras foram colocadas em um recipiente de vidro esterilizado para a preparação da alcoolatura, permanecendo submersas em álcool por um período de 72 horas. Das folhas do Melão de São Caetano e do farelo de batata de purga foram utilizados $1000 \mathrm{~g}$ em $6000 \mathrm{~mL}$ de álcool etílico PA e $300 \mathrm{~g}$ em $4.300 \mathrm{~mL}$ de álcool etílico PA, respectivamente.

Após esse período, procedeu-se à filtragem das mesmas, utilizando papel de filtro e o líquido filtrado (volume inicial $\left.=V_{1}\right)$, foi transferido em pequenas porções $(300 \mathrm{~mL})$ para um balão de $1000 \mathrm{~mL}$ para a obtenção do extrato liquido, a uma temperatura de $40 \pm 50^{\circ} \mathrm{C}$. O extrato obtido (volume final $=\mathrm{V}_{2}$ ), foi transferido para recipiente de vidro de cor âmbar, colocado à temperatura ambiente por 10 minutos e, em seguida, mantido sob refrigeração até o momento em que os animais receberiam o tratamento. A concentração da planta em $\mathrm{g} / \mathrm{mL}(\mathrm{m} / \mathrm{v})$, do extrato obtido a partir das folhas do melão de São Caetano ( $M$. charantia) calculada foi de $2,17 \mathrm{mg} / \mathrm{mL}$, o volume final $\left(\mathrm{V}_{2}\right)$ após a extração do álcool foi $460,2 \mathrm{~mL}$ e a dose utilizada correspondeu a $2,7 \mathrm{~mL} / \mathrm{Kg}$ de peso corpóreo. A concentração do extrato obtida a partir do farelo da batata purga (O. hamiltonii) foi $0,7 \mathrm{mg} / \mathrm{ml}, \mathrm{o} \mathrm{V}_{2}$ após extração do álcool foi $450 \mathrm{~mL}$ e a dose utilizada foi 0,56 $\mathrm{mL} / \mathrm{kg}$ de peso corpóreo por via enteral.

\section{Teste de redução da contagem de OPG nas fezes}

As médias aritméticas da contagem do número de ovos por grama de fezes, para cada grupo tratado (OPGt), foram calculadas e comparadas com as médias contadas no grupo controle (OPGc). A redução na contagem de ovos por grama de fezes (RCOF) foi determinada, usando a fórmula descrita por Coles et al. (1992):

\section{RCOF=[1-(OPGt/OPGc) $] \mathbf{x 1 0 0}$}

Em que:

$\mathrm{RCOF}=$ teste de redução da contagem de ovos por grama de fezes

$\mathrm{OPGt}=$ média do número de ovos por grama de fezes do grupo de animais tratados

$\mathrm{OPGc}=$ média do número de ovos por grama de fezes do grupo controle

\section{Delineamento Experimental}

Os animais foram separados em quatro grupos, sendo cada grupo composto por 10 caprinos. O GRUPO 1- animais controle negativo, tratados com água destilada; o GRUPO 2 animais tratados com o extrato alcoólico da $O$. hamiltonii (batata de purga), na dose de $0,56 \mathrm{~mL} / \mathrm{kg}$ de peso corpóreo; o GRUPO 3 - os animais tratados com o extrato alcoólico da $M$. charantia (melão de São Caetano), na dose de $2,7 \mathrm{~mL} / \mathrm{Kg}$ de peso corpóreo e no GRUPO 4 - animais controle positivo tratados com anti-helmíntico à base de moxidectina $0,2 \%$, administrado oralmente, em dose única de $1,0 \mathrm{~mL} / 10 \mathrm{Kg}$. Todos os grupos tratados com extrato vegetal receberam essa dose por três dias consecutivos, uma vez ao dia

O monitoramento da infecção helmíntica foi feito por meio da determinação do número de OPG dos animais, realizado mensalmente. Os resultados obtidos com a contagem de OPGs foram tratados pela fórmula $\log (\mathrm{x}+1)$ e submetidos à análise de variância e teste de Tukey a 5\% de significância.

\section{RESULTADOS E DISCUSSÃO}

Com a metodologia utilizada, os resultados obtidos demonstraram uma redução na contagem do número do número de OPG de fezes nos animais tratados em relação ao grupo controle negativo (Tabela 1).

Foi observada uma redução no OPG dos animais dos Grupos G2, G3 e G4. A redução (Tabela 01) variou de $40 \%$ a $100 \%$ e de $90 \%$ a $100 \% 30$ e 60 dias após o tratamento, respectivamente. Ressaltando-se ainda que nenhum efeito colateral foi observado nos animais dos grupos tratados (Tabela 1).

Para o grupo (G2) tratado com o extrato de batata de purga $(O$. hamiltonii), a redução média no OPG foi $63 \%$ observado 30 dias pós-tratamento e $90 \%$ observado 60 dias pós-tratamento (Tabela1). A análise dos dados mostrou que houve diferenças significativas ao nível considerado na redução de OPG nos 30 e 60 dias pós-tratamento $(\mathrm{P}>0,05)$. Comparado com o G1 a média do percentual de redução do OPG, aumentou para $77 \%$ e $95 \%$, respectivamente. Dados que diferem daqueles informados por Rodrigues et al. (2007) 
que obtive com extrato aquoso de batata-de- purga nas fêmeas e machos, 21 dias pós tratamento de 49,4 e 50\% de eficácia, respectivamente e por Silva et al. (2010) que obteve redução de $70 \%$ utilizando farelo de batata-de-purga em caprinos. Segundo preconiza o Grupo Mercado Comum GMC (1996), o uso do extrato de batata de purga obtive uma redução significativa no número de OPG em caprinos.

No grupo tratado com o extrato do melão de São Caetano (M. charantia), foi observada uma redução média no OPG de $40 \%$ nos dias 30 e 60 pós-tratamento, valores esses que não diferiram significativamente $(\mathrm{P}>0,05)$. Esses resultados não corroboram com os relatos de Almeida et al. (2007), que utilizando folhas de M. charantia em caprinos naturalmente infectados obteve uma redução no número de OPG de $82 \%$ visto aos 60 dias pós-tratamento. Quando comparados aos do grupo G1 no mesmo período estudado os valores obtidos, expressou um aumento na redução para $49 \%$ e $56 \%$ para 30 e 60 dias pós-tratamento, respectivamente. Ao se comparar esses resultados com o que preconiza o GMC (1996), os resultados obtidos com o uso do extrato do melão de São Caetano (M. charantia) expressa uma redução no de OPG, pouco significativa.
O grupo tratado com a moxidectina a $0,2 \%$, apresentou uma redução no OPG de 100\%, 30 e 60 dias póstratamento. A análise dos resultados mostrou diferenças significativas $(\mathrm{P}>0,05)$ ao nível considerado. Esses resultados não corroboram com os apresentados por Papadopoulos et al. (2004) que, em condições experimentais semelhantes, registraram uma redução de $93,5 \%$ no número de OPG em fezes de caprinos tratados com a moxidectina $0,2 \%$ por via oral 56 dias pós-tratamento. Rodrigues et al. (2007) em condições experimentais semelhantes, avaliando a sensibilidade de nematóides gastrintestinais de caprinos em propriedades do sistema produtivo de caprinos do Sertão Paraibano, registrou uma redução no número de OPG de $89,9 \%$ aos 21 dias pós-tratamento. Os resultados obtidos indicaram que a moxidectina a $0,2 \%$ apresentou uma ação farmacológica satisfatória na redução do OPG, segundo preconiza o GMC (1996).

Durante o período de estudo, observou-se que de todas as amostras coletadas $100 \%$ estavam positivas (Tabela 2) para helmintos da superfamília Trichostrongyloidea.

Tabela 1 - Percentual de eficiência de extratos vegetais e moxidectina a $0,2 \%$ sobre a redução no número de ovos por grama de fezes de caprinos naturalmente infectados, por nematóides gastrintestinais, em amostras coletadas 30 e 60 dias pós-tratamento.

\begin{tabular}{lccccccc}
\hline Tratamentos & $\begin{array}{c}\text { OPG } \\
\text { Dia zero }\end{array}$ & $\begin{array}{c}\text { OPG } \\
\text { Dia 30 }\end{array}$ & $\begin{array}{c}\text { OPG } \\
\text { Dia 60 }\end{array}$ & Redução de OPG (\%) & \multicolumn{2}{c}{ Em relação ao Grupo 1 } \\
\hline G1 & & & & 30 dias & 60 dias & 30 dias & 60 dias \\
G2 & $4623^{\mathrm{ab}}$ & $5173^{\mathrm{ab}}$ & $6000^{\mathrm{ab}}$ & -12 & -30 & & \\
$\mathrm{G} 3$ & $3160^{\mathrm{b}}$ & $1170^{\mathrm{c}}$ & $319^{\mathrm{d}}$ & 63 & 90 & 77 & 95 \\
$\mathrm{G} 4$ & $4394^{\mathrm{ab}}$ & $2622^{\mathrm{bc}}$ & $2620^{\mathrm{bc}}$ & 40 & 40 & 49 & 56 \\
\hline
\end{tabular}

G1 - água destilada; G2 - batata de purga; G3 - Melão de São Caetano; G4 - moxidectina a 0,2\%. Letras comparam as médias entre linhas. Letras diferentes indicam diferença significativa $(\mathrm{p}<0,05)$.

Tabela 2 - Percentual de gêneros de helmintos em coproculturas de caprinos naturalmente infectados, por nematóides gastrintestinais, em amostras coletadas 30 e 60 dias pós-tratamento com extratos vegetais e moxidectina a $0,2 \%$

\begin{tabular}{|c|c|c|c|c|c|c|c|c|c|c|c|c|}
\hline & \multicolumn{3}{|c|}{ Grupo 1} & \multicolumn{3}{|c|}{ Grupo 2} & \multicolumn{3}{|c|}{ Grupo 3} & \multicolumn{3}{|c|}{ Grupo 4} \\
\hline Gêneros & 0 & 30 & 60 & 0 & 30 & 60 & 0 & 30 & 60 & 0 & 30 & 60 \\
\hline Bunostomun & $18 \%$ & $17 \%$ & $18 \%$ & $10 \%$ & $10 \%$ & $14 \%$ & $14 \%$ & $8 \%$ & $14 \%$ & $11 \%$ & $0 \%$ & $0 \%$ \\
\hline Haemonchus & $57 \%$ & $59 \%$ & $58 \%$ & $62 \%$ & $34 \%$ & $36 \%$ & $56 \%$ & $42 \%$ & $41 \%$ & $61 \%$ & $0 \%$ & $0 \%$ \\
\hline Oesophagostomun & $8 \%$ & $7 \%$ & $9 \%$ & $6 \%$ & $42 \%$ & $36 \%$ & $9 \%$ & $37 \%$ & $33 \%$ & $3 \%$ & $0 \%$ & $0 \%$ \\
\hline Trichostrongylus & $17 \%$ & $18 \%$ & $16 \%$ & $22 \%$ & $14 \%$ & $14 \%$ & $21 \%$ & $15 \%$ & $12 \%$ & $25 \%$ & $0 \%$ & $0 \%$ \\
\hline
\end{tabular}

Grupo 1 - água destilada; Grupo 2 - batata de purga; Grupo 3 - melão de são caetano; Grupo 4 - moxidectina a 0,2\%. 
O Haemonchus foi o gênero mais prevalente, corroborando com resultados obtidos em experimentos realizados em rebanhos caprinos no estado do Ceará e em outros estados no nordeste brasileiro (VIEIRA; CALVACANTI, 1998; RODRIGUES et al., 2007). Está persistência do gênero Haemonchus, provavelmente, se deva ao seu alto potencial biótico, que contribui para que a resistência, nesse gênero, se desenvolva de forma rápida e efetiva (ECHEVARRIA; TRINDADE, 1989). Aliado a esse potencial, o gênero possui uma grande variabilidade genética e, possivelmente, alberga o alelo que causa a diminuição da susceptibilidade a uma droga (BLACKHALL et al., 1998).

\section{CONCLUSÕES}

A partir da metodologia empregada neste estudo, com caprinos da mesorregião do sertão paraibano e nas condições a que foram submetidos, os resultados obtidos sugerem que:

- O uso do extrato alcoólico de batata de purga (Operculina hamiltonii) e das folhas de melão de são Caetano (Momordica charantia) são eficazes na redução do número de ovos por grama de fezes (OPG) de helmintos gastrintestinais em caprinos naturalmente infectados, representando uma alternativa no controle desses parasitos.

- O gênero Haemonchus é o mais prevalente dentre os demais gêneros da superfamília Trichostrongyloidea em infecções helmínticas naturais de caprinos no semiárido paraibano.

\section{REFERÊNCIAS BIBLIOGRÁFICAS}

ALMEIDA, M.A.O. de et al. Efeitos dos extratos aquosos de folhas de Cymbopogon citratos (DC) Stapf (Capim Santo) e Digitaria insularis (L) Fedde (CapimAçu) sobre cultivo de larvas de nematóides gastrintestinais de caprinos. Revista Brasileira de Parasitologia, São Paulo, v.12, n.3, p.125-129, 2003.

ALMEIDA, W.V.F. et al. Avaliação de plantas medicinais em caprinos da região do Semi-Árido Paraibano naturalmente infectados por nematóides gastrintestinais. Caatinga, Mossoró, v.20, n.3, p.1-7, 2007.

ANUALPEC. Anuário da pecuária brasileira. São Paulo: Consultoria \& Comércio, 2002. 400p.

ARAÚJO-LIMA, R.C.A. et al. Difusão do uso de plantas medicinais com ação antiparasitária: uma alternativa para o controle da verminose de caprinos e ovinos na região semi-árida da Paraíba. In: CONGRESSO BRASILEIRO DE

\section{EXTENSÃO UNIVERSITÁRIA, 1.; ENCONTRO NACIONAL INSTITUCIONAL DE EXTENSÃO UNIVERSITÁRIA, 2.; FEIRA UNIVERSIDADE E SOCIEDADE, 1., 2002, João Pessoa. Resumos... João Pessoa: COPREX/UFPB, 2002. p.378.}

BLACKHALL, W.J. et al. Haemonchus contortus: selection at a glutamate-gated chloride channel gene in ivermectin- and moxidectin-selected strains.

Experimental Parasitology, v.90, p.42-48, 1998.

CEZAR, A.S.; CATTO, J.B.; BIANCHIN, I. Controle alternativo de nematódeos gastrintestinais dos ruminantes: atualidade e perspectivas. Ciência Rural, Santa Maria, v.38, n.7, p.2083-2091, out. 2008.

ECHEVARRIA, F.A.M.; TRINDADE, G.N.P. Anthelmintic resistance by Haemonchus contortus to ivermectin in Brazil. Veterinary Record, v.124, p.147-148, 1989.

GOPAL, R.M.; POMROY, W.E.; WEST, D.M. Resistance of field isolates of Trichostrongylus colubriformis and Ostertagia circumcincta to ivermectin. International Journal of Parasitology, Lancaster, v.29, p.781-786, 1999.

GORDON, H.M.; WHITLOCK, H.V. A new technique for counting nematode eggs in sheep faeces. Journal Council Science Industry Research of Australia, Melbourne, v.12, p.50-52, 1939.

\section{GRUPO MERCADO COMUM. Regulamento técnico para registros de produtos antiparasitários de uso veterinário: decisão n. 4/91, resolução n. 11/93, Mercosul, resolução n. 76, 1996. Brasília, 1996.}

KEITH, R.K. The differenciation of the infective larvae of some common nematode parasites of cattle . Australian Journal Zoollogia, Melbourne, v.35, n.1, p.223-253, 1953.

KRECEK, R.C.; WALLER, P.J. Towards the implementation of the "basket of options" approach to helminth parasite control of livestock: Emphasis on the tropics/subtropics. Veterinary Parasitology, v.139 p.270-282, 2006.

MCLEOD, R.S. The economic impact of worm infections in small ruminants in Southeast Asia, India and Australia. In: SANI, R.A.; GRAY, G.D.; BAKER, R.L. (Eds.). Worm control for small ruminants in tropical Asia. [S.1.]: ACIAR, 2004. p.23-33. 
MOLENTO, M.B. Resistência de helmintos em ovinos e caprinos. Revista Brasileira de Parasitologia

Veterinária, v.13, n.1, 2004. Suplemento.

PAPADOPOULOS, E. et al. Evaluation of the efficacy of moxidectin in a herd of goats after long-standing consistent use. Small Ruminant Research, v.57, p.271-275, 2004.

ROBERTS, F.H.S.; O’SULLIVAN, J.P. Methods for egg counts and larval cultures for strongyles infesting the gastro-intestinal tract of cattle. Australin Agriculture Research, Melbourne, n.1, p.19, 1950.

RODRIGUES, A.B. et al. Sensibilidade dos nematóides gastrintestinais de caprinos a anti-helmínticos na mesorregião do Sertão Paraibano. Pesquisa Veterinária Brasileira, Rio de Janeiro, v.27, n.4, p.162-166, 2007.
ROEDER, R. Promoção da agricultura em regiões semiáridas do Nordeste (Piauí) brasileiro: pesquisa sobre a pecuária nos planaltos da chapada. Teresina: DNOCS, 1988. 125 p.

SILVA, C.F. et al. Avaliação da eficácia de taboa (Typha domingensis Pers.) e batata-de-purga [Operculina hamiltonii (G. Don) D.F. Austin \& Staples] in natura sobre nematóides gastrintestinais de caprinos, naturalmente infectados, em clima semi-árido. Revista Brasileira de Plantas Medicinais, Botucatu, v.12, n.4, p.466-471, 2010.

VIEIRA, L.S.; CAVALCANTE, A.C.R. Resistência antihelmíntica em nematóides gastrintestinais de caprinos. Revista Brasileira de Medicina Veterinária, v.20, n.3, p.112-117, 1998. 